\title{
Comparing various dose of PGE2 gel in induction of labour-maternal and fetal outcome
}

\author{
Maya Menon*, Sridevi TA, Vasantha Krishnamurthy
}

Department of Obstetrics and Gynaecology, ESIC Medical College and PGIMSR, Chennai, Tamilnadu, India

Received: 02 March 2016

Accepted: 30 March 2016

\section{*Correspondence:}

Dr. Maya Menon,

E-mail: mayasree@gmail.com

Copyright: $\odot$ the author(s), publisher and licensee Medip Academy. This is an open-access article distributed under the terms of the Creative Commons Attribution Non-Commercial License, which permits unrestricted non-commercial use, distribution, and reproduction in any medium, provided the original work is properly cited.

\section{ABSTRACT}

Background: Induction of labour is the artificial initiation of labour before spontaneous onset of labour sets in. The frequency of induction varies by location and institution. The objective of this study was to study the maternal and fetal outcome after induction using different doses of PGE2 intracervical gel.

Methods: A retrospective study done at E.S.I .C Medical College and PGIMSR, K. K. Nagar Chennai, Tamilnadu, India using data of deliveries conducted during the period November 2013 to October2014.

Results: Total number of deliveries during the study period was 847 , and the total numbers of cases induced were 105. Out of these patients who had undergone induction 39 received one dose, 40 received two doses and 26 received three doses in 24 hours at 6 hours interval. There was significant difference in mode of delivery $(\mathrm{p}=0.000)$, change in Bishop Score ( $\mathrm{p}=0.009)$, NICU admission ( $\mathrm{p}=0.003)$ between the three groups.

Conclusions: Our data proves that the maternal and fetal outcomes were better with one or two doses of PGE2 gel. Third dose of PGE2 gel unnecessarily prolonged the duration of labour leading to increased emergency caesarean section and increased neonatal intensive care unit admissions (NICU).

Keywords: Induction of labour, PGE2 gel, Mode of delivery

\section{INTRODUCTION}

Induction of labour is the artificial initiation of labour before spontaneous onset of labour sets in. The frequency of induction varies by location and institution. ${ }^{1}$ In the interest of mother or baby or both it becomes necessary to interrupt the pregnancy. ${ }^{2}$ Thus elective induction of labour at term have increased because ultra-sonogram, Doppler and medical conditions in mother warrant an urgent termination of pregnancy. PGE2 intracervical gel application is the safest mode used for induction of labour in unscarred uterus for an unfavourable cervix. Prostaglandins cause alteration of extracellular ground substance of cervix by increasing collagenase, elastase, glycosaminoglycan's, and dermatan sulphate and hyaluronic acid levels. ${ }^{3}$ Relaxation of smooth muscle of cervix and formation of gap junctions lead to initiation of uterine contractions. ${ }^{4,5}$ As per the recommended protocol,
$0.5 \mathrm{mg}$ per dose is kept endocervical up to a maximum of 3 doses 6 hours apart. The goal of induction is to achieve a successful vaginal delivery. Induction to delivery interval has been decreased dramatically by introduction of prostaglandin, and associated complication of fetal infection and amnionitis. ${ }^{6}$ Many studies have compared the effect of PGE2 gel and PGE1vaginal tablet for induction of labour at term. Prostaglandins have been shown to induce cervical ripening and to stimulate uterine contractions and are effective in numerous clinical trial however not many studies exist which investigate the effect of third dose of PGE2 gel in induction of labour. ${ }^{7}$ Thus, we planned to study the maternal and fetal outcomes with PGE2 intracervical gel with one, two and three doses for induction of labour. 


\section{METHODS}

This is a retrospective study done at ESIC Medical College K. K. Nagar, Chennai, Tamilnadu, India. The Medical records of the total number of deliveries and caesarean sections during the period from November 2013 to October 2014, was collected from medical records department.

From the records, the cases with singleton pregnancies, cephalic presentation, bishop score of five or less, AFI 5 or more, reactive FHR pattern, post-dated pregnancy, membrane intact / ruptured were included. The cases with the following criteria were excluded from the study, para three or more, previous LSCS, abnormal fetal heart rate tracing, hypersensitivity to prostaglandin. The use of PGE2 gel by any route has been reported to improve vaginal delivery, initially PGE2 gel was considered to be unsafe and dangerous in females of high parity but now studies have reported it to be safe and effective method in grand multiparous women. ${ }^{8}$

The objective was to assess the progress of labour, to assess the mode of delivery and to study NICU admission. As per the protocol the data showed $0.5 \mathrm{mg}$ of PGE2 gel intracervical was instilled, the dose was repeated if necessary every 6 hours maximum of 3 doses in 24 hours. ${ }^{1}$ Pelvic examination findings were recorded from the data. The details of labour augmentation with oxytocin in patients who had poor uterine contraction and the continuous electronic fetal heart rate monitoring were collected.

Out of the 105 induced, 39 had received one dose of cerviprime (group I), 40 received two doses (group II), and 26 received 3 doses (group III). Labour induction was considered successful if vaginal delivery occurred. A primary outcome was the interval from first dose of the drug to vaginal delivery.

The variables in maternal outcome include Labour induction, change in bishop score, time interval from induction to onset of adequate uterine contractions (3 contractions in 10 minutes), mode and route of delivery, Indications for LSCS, number of Emergency LSCS performed for abnormal FHR pattern, number of doses of PGE2 gel used, oxytocin augmentation. ${ }^{4}$

Adverse effects such as uterine contraction abnormalities, FHR pattern abnormalities and specific prostaglandin side effects such hyperpyrexia, vomiting and diarrhoea, incidence of postpartum haemorrhage, cervical tears, and vaginal tears were noted.

The variables in neonatal outcome include birth weight, Apgar score at 1 and 5 minutes, incidence of meconium stained amniotic fluid, admission to neonatal intensive care unit.
Statistical Analysis of data was performed using SPSS version 16, variables were analyzed with Chi square test, Fischer exact $t$ test. The $\mathrm{p}$ value $<0.05$ was considered as significant.

\section{RESULTS}

Out of the total 847 deliveries conducted in the institution between November 2013 and October 2014, 105 was induced, 302 had spontaneous delivery, 100 had undergone elective repeat LSCS, 40 had undergone elective LSCS, 200 had Emergency LSCS and 100 had emergency repeat LSCS.

Of the 105 eligible women included for final analysis 39 received one dose (group I), 40 received two dose (group II) and 26 received three doses (group III) at 6 hours interval within 24 hours.

Labour augmentation with oxytocin was required for 7 patients in group III $(n=26)$, out of which 4 delivered normally and 3 patients had undergone emergency caesarean section, indication being fetal distress.

The descriptive statistics of the study population is given in (Table 1). There was no significant difference in the baseline data of the study population with gestational age, parity, BMI, AFI, bishop score.

Table 1: Descriptive statistics of the study population.

\begin{tabular}{|lll|}
\hline Parameters & Mean \pm SD & Range \\
\hline Age & $26.1 \pm 3.7$ & $19-38$ \\
\hline Gestational age & $38.9 \pm 2.0$ & $36-40$ \\
\hline Height & $154.9 \pm 13.8$ & $135-172$ \\
\hline Weight & $55.9 \pm 10.1$ & $52-112$ \\
\hline BMI & $22.9 \pm 3.7$ & $19.4-40.8$ \\
\hline Amniotic fluid index & $8.7 \pm 2.6$ & $8.1-15.4$ \\
\hline Bishop score & $3.5 \pm 1$ & $2-5$ \\
\hline Cervical length & $1.2 \pm 0.5$ & $2.5-3.5$ \\
\hline
\end{tabular}

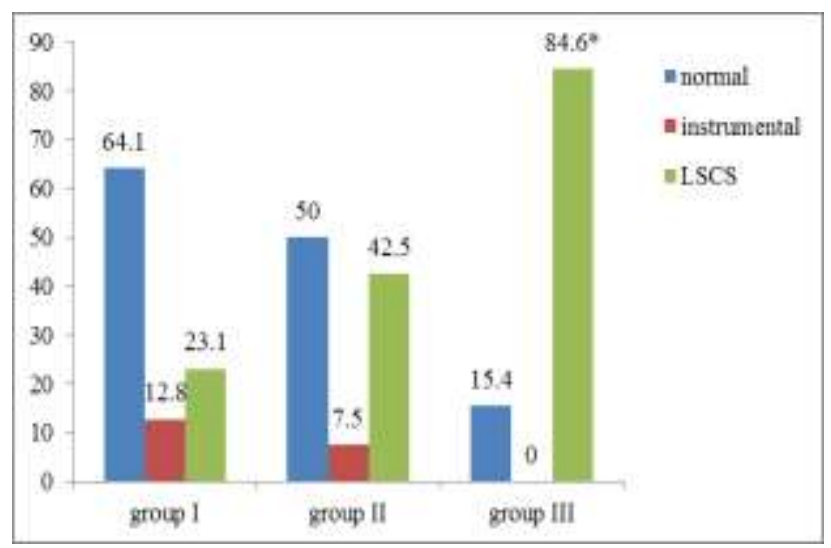

Figure 1: Mode of delivery and percentage distribution of patients in the three groups. 
The mean induction delivery interval was 8.3 hours, 23.9 hours and 32.6 hours in group 1, II and III respectively. On analysing the change in the bishop score between the three groups it was found that even at a bishop's score of 4-5 all the patients went in for caesarean section in group III. In group I, 19 had vaginal delivery while 4 had LSCS and in group II 4 patients had vaginal, 7 had LSCS (Table 2). The mean induction delivery interval and the percentage distribution of patients in the three groups are shown in (Table 3 ). The induction delivery interval was significantly higher in group III $(\mathrm{p}<0.001)$.
Table 2: Relation between Bishop Score and mode of delivery.

\begin{tabular}{|lcllll|}
\hline $\begin{array}{l}\text { Bishop score } \\
\text { Mode of } \\
\text { delivery }\end{array}$ & Vaginal & LSCS & Vaginal & LSCS \\
\hline Group I & $11(28.2)$ & $5(12.8)$ & $19(8.7)$ & $4(10.3)$ \\
\hline Group II & $19(47.5)$ & $10(25)$ & $4(10)$ & $7(17.5)$ \\
\hline Group III & $4(15.4)$ & $14(53.9)^{*}$ & 0 & $8(30.7)^{*}$ \\
\hline
\end{tabular}

* $\mathrm{p}=0.009$ (highly significant).

Table 3: Mean induction delivery interval between the three groups.

\begin{tabular}{|c|c|c|c|c|c|}
\hline \multirow{3}{*}{ Group } & \multicolumn{5}{|c|}{ Induction delivery interval } \\
\hline & $\leq \mathbf{2 4}$ hours & $\leq \mathbf{2 4}$ hours & $>24$ hours & $>24$ hours & Total \\
\hline & Mean \pm SD & $\mathrm{N}(\%)$ & Mean \pm SD & $\mathrm{N}(\%)$ & $\mathrm{N}$ \\
\hline I & $7.63 \pm 3.1$ & $39(100)$ & 0 & 0 & 39 \\
\hline II & $16.55 \pm 0.8$ & $27(67.5)$ & $38.53 \pm 9.9$ & $13(32.5)$ & 40 \\
\hline III & $18.25 \pm 2.4$ & $10(38.5)$ & $47.4 \pm 2.9^{*}$ & $16(61.5)$ & 26 \\
\hline
\end{tabular}

${ }^{*} \mathrm{p}<0.000$ significance (highly significant).

Table 4: Neonatal intensive care unit admission in vaginal and LSCS deliveries.

\begin{tabular}{|lllllll|}
\hline No of doses & \multicolumn{2}{l}{ NICU admissions in LSCS $(\mathbf{n = 4 8 )}$} & NICU admissions in vaginal/instrumental delivery $(\mathbf{n = 5 7 )}$ \\
\hline I & $\mathbf{N}(\boldsymbol{\%})$ & $\mathbf{N}(\boldsymbol{\%})$ & Total $(\mathbf{N})$ & $\mathbf{N}(\boldsymbol{\%})$ & $\mathbf{N}(\boldsymbol{\%})$ & Total $(\mathbf{N})$ \\
\hline II & $2(22.2)$ & $7(77.8)$ & 9 & $2(6.7)$ & $28(93.3)$ & 30 \\
\hline III & $5(29.4)$ & $12(70.6)$ & 17 & $2(8.7)$ & $21(91.3)$ & 23 \\
\hline
\end{tabular}

* $\mathrm{p}=0.033$ significance.

Figure 1 depicts the mode of delivery in the three groups and the percentage of patients with normal, instrumental and LSCS. There is a significant difference in the mode of delivery in the three groups with more number of LSCS in group III. The most common indication for LSCS was failed induction $71 \%(\mathrm{p}=0.016)$, followed by CPD $67 \%$, fetal distress $31 \%$, Non progress of labour $25 \%$ in group III. Table 4 shows the number of NICU admissions of the neonate delivered by vaginal and LSCS. There were statistical significant NICU admissions in group III. The incidence of abnormal cardiograph was $23 \%, 31 \%$ and $46 \%$ in the three groups respectively. There were 10 cases of maternal haemorrhage in group III, 4 in group II and 1 in group I.

\section{DISCUSSION}

PGE2 gel is the preferred pharmacological method of induction of labour. PGE2 acts on the cervix by dissolving the collagen structural network of the cervix. Fetal membranes and decidua produce PGE2 during pregnancy and labour. Release of this hormone leads to changes in the biochemistry of the cervix and also stimulates the production of PGF $2 \alpha$. In turn, PGF $2 \alpha$ sensitizes the myometrium to oxytocin. Exogenous administration of PGE2 is known to mimic this natural process and lead to cervical ripening or labour. ${ }^{9}$ Advantage of PGE2 include patient acceptance, a lower operative rate than oxytocin, and less need for oxytocin augmentation when used with an unfavorable cervix. ${ }^{10}$ The present study of comparison of various doses of PGE2 and its effect on maternal and fetal outcome shows that vaginal delivery and instrumental delivery was more in one and two doses of PGE2 whereas out of the 26 patients who were given 3 doses of PGE2, 22 had to undergo Emergency LSCS. Not many studies exist that have compared the maternal and fetal outcome using three doses of PGE2 gel. Studies have compared the efficacy between PGE2 and PGE1 or PGE2 and Foley's catheter. The study was planned to understand whether there was an undue delay in delivery with the standard protocol of PGE2 gel. ${ }^{2}$

Various studies have shown the beneficial effects of intracervical PGE2 gel in improving bishop score, the improvement ranged from 3 to 7 points. ${ }^{11}$ The success of induction of labour was found to be directly proportional to the Bishop score at instillation. Assessment of cervical status is fundamental for the clinician to estimate the likelihood of a successful vaginal delivery. Of the bishop score criteria for predicting successful induction, the most important is cervical dilatation, followed by effacement, 
station and position, with the least important being consistency.

An Indian study done in comparison between PGE1 and PGE2 found that PGE2 group took 11.5 hours. Whereas our study shows a mean induction delivery interval of 20.2 hours. The difference in the mean induction delivery interval may be due to the initial bishop score which was 4 in their study whereas it was 2 in our study. ${ }^{2}$

Studies have observed a significant difference between parity, mean induction time interval and PGE2 gel but their data does not state the number of doses gel required for induction. Our results show that $29 \%$ of PRIMI, $18 \%$ of $2^{\text {nd }}$ gravida, and none of multipara required 3 rd dose of PGE2gel. The overall mean induction to delivery time in our study was 20.2 hours. Various studies have shown considerable variation as far as induction delivery time is concerned ranging from 9 hours Noah et al to 17.9 hours Thiery et al. ${ }^{12-15}$

Our data on secondary outcome variables showed that there was no significant association in NICU admission for patients who underwent LSCS in group III. Kavita et al observed that significant number of babies required NICU admission with PGE2 gel, though there was no particular mention regarding the number of doses. ${ }^{17}$

Following the protocol of 3 doses in 6 hours interval the number of LSCS was $19 \%$ in group I, 35\% in group II, and $46 \%$ in group III in our study. Warke et al in his study has observed that the number of LSCS was extremely high with PGE2 gel instillation 2nd dose after 24 hours. ${ }^{4}$

The commonest indication for LSCS was found to be failed induction and fetal distress. Similar results have been observed by Deodhar $\mathrm{P}$ et al and Lara. We also observed that $10(38.6 \%)$ out of 26 patients in Group III had post-partum haemorrhage. ${ }^{12,16}$

Thus we emphasize that waiting for the progress of labour beyond 2 nd dose will lead to increase in maternal and fetal morbidity. As the maternal and fetal outcome shows that there was increased rate of LSCS and NICU admission in group III. Is it worth to recommend the third dose of PGE2 gel for induction of labour? The benefit of induction is in the hands of the clinician to decide the duration of labour and to achieve a successful vaginal delivery with less maternal and fetal morbidity.

Similar study done on a larger population, may throw a better vision on the usage of third dose of PGE2gel.

\section{CONCLUSIONS}

Induction of labour confers benefit in both maternal and fetal outcome. It can be a costly affair when cervix is unfavourable for delivery. Agent of choice is PGE2 and appears to be a perfect substitute to oxytocin. In this study, comparing the three groups, who received one, two and three doses of PGE 2 gel intracervical instillation, group I, II patients had more number of vaginal deliveries and group III had increased number of LSCS and neonatal admission. Thus the study proves that induction of labour with PGE2 gel with one and two doses shows better maternal and fetal outcome.

Funding: No funding sources

Conflict of interest: None declared

Ethical approval: The study was approved by the Institutional Ethics Committee

\section{REFERENCES}

1. Dean L, Anne B. Induction of labour. J Obstet Gynaecol Can. 2013;35(9)S1-17.

2. Girish S, Manjunath AP. A randomized controlled trial comparing low dose vaginal misoprostol and dinoprostone gel for labour induction. JOGI. 2011;153-60.

3. Ulmstein, Wingerup L, Anderson. Comparison of prostaglandin E2 gel and intravenous oxytocin for induction of labour. Obstet Gynecol. 1979;54:581-4.

4. Warke HS, Sarogi RM, Sanjawalla SM. Prostaglandin E2 gel in ripening of cervix in induction of labour. J Postgrad Med. 1999;45:105-7.

5. Maillot KV, Stunhsatz HW, Mohanaradhakrishnan V. Changes in glycosaminoglycan distribution in the human uterine cervix during pregnancy and labour. Am J Obstet Gynecol. 1979;135:503-6.

6. Parmar M, Aherwar R, Jahan I. Comparative study of $25 \mathrm{mg}$ vaginal misoprostol versus cerviprime gel for induction of labour at term. Int $\mathrm{J}$ Reprod Contracept Obstet Gynecol. 2014;3(4):887-92.

7. Nayak S, Pati T, Sahu N, Venkatarao, Sahu MC, Marandi S. Role of Intracervical dinoprostone gel administration versus vaginal administration of oral prostaglandin E2 tablet for induction of labour. Int $\mathrm{J}$ Pharm Sci Rev Res. 2015;30(1):279-84.

8. Sharma A, Monika S, Sahay PB, Upadhyaya SK, Thaddanee R, Khilnan AK. A comparative study of prostaglandin E2 gel with oxytocin infusion for induction of labour. NJIRM. 2013;4(6):111-5.

9. George M, George MS. Effect of cerviprime on cervical ripening and induction of labour. JEMDS 2015;457:3162-8.

10. Patil P, Patil A. Misoprostol versus cerviprime gel for induction of labour. IJMRR. 2013;1(2):63-8.

11. Trofatter KF, Bowers D, Gall DA. Preinduction cervical ripening with prostaglandin E2 (Prepidil) gel. Am J Obstet Gynecol. 1985;153:268-71.

12. Deodhar P, Rai S. Comparative study of misoprostol versus dinoprostone for induction of labour. Indian Medical Gazette. 2013;454-8.

13. Anand AK, Mir S. A randomised comparison between intravaginal misoprostol and inracervical dinoprostone for cervical ripening and labour induction in participants with unfavourable cervices. Jammu and Kashmir Science. 2012;14:115-9. 
14. Noah ML, Decoster JM, Frase W. Preinduction cervical softening with endocervical PGE2 gel. A multicentric trial. Acta Obstet Gynecol Scand. 1987;66:3-7.

15. Thiery M, Decoster JM, Parewijck W. Endocervical prostaglandin E2 gel for preinduction cervical softening. Clin Obstet Gynecol. 1984;27:429-39.
16. Pullen LC, Jacqueline A. Labour induced by foley catheter versus Prostaglandin E2. Medscape Medical News. 2011;752159.

17. Kavita A, Achla B, Aruna B, Abha A. Randomized comparison of isosorbide mononitrate and pge2 gel for cervical ripening at term including high risk pregnancy. International Journal of Reproductive Medicine. 2014;147274 -9.

Cite this article as: Menon M, Sridevi TA,

Krishnamurthy V. Comparing various dose of

PGE2 gel in induction of labour-maternal and fetal outcome. Int J Reprod Contracept Obstet Gynecol 2016;5:1520-4. 